\title{
Fatal Necrotising Soft Tissue Infection Caused by Escherichia coli in Alcoholic Liver Cirrhosis
}

\author{
MIO KURIMOTO ${ }^{1}$, Mahoko Ikeda ${ }^{1}$, Ichiro Hirayama ${ }^{1}$, Yatsuya Kobayashi ${ }^{1}$, Mio Shikama $^{1}$, \\ Hitomi Nakamura ${ }^{1}$, Yuji Wakimoto ${ }^{1}$, Oshiro Yohei ${ }^{1}$, Miyuki Yamamoto ${ }^{1}$, Naoki Hayase ${ }^{1}$, \\ Takahiro Hiruma ${ }^{1}$, Shu Okugawa ${ }^{1}$, Kent Doi ${ }^{1}$, Naoto Morimura ${ }^{1}$, and Kyoji Moriya ${ }^{1}$ \\ ${ }^{1}$ The University of Tokyo Hospital
}

May 31, 2021

\begin{abstract}
NSTI caused by E. coli monomicrobial infection is an extremely severe condition with high mortality. This report presented a case of monomicrobial NSTI caused by E. coli with septic shock in a patient with alcoholic liver cirrhosis and mentioned both pathogen virulence factors and host susceptibility factors.
\end{abstract}

\section{Introduction}

Necrotising soft tissue infections (NSTIs) can be defined as infections of any of the layers within the soft tissue, comprising the dermis, subcutaneous tissue, superficial fascia, deep fascia, or muscle. The mortality rate for patients with these infections is high, even in cases of invasive surgical treatment and adequate antibiotic therapy (1). The NSTIs are classified into three types: type 1 is a polymicrobial infection and is the most common form of NSTI; type 2 is a monomicrobial infection caused by Streptococcus pyogenes orStaphylococcus aureus ; and type 3 is a monomicrobial infection caused by gram-negative bacilli such as Vibrio spp . (2). Escherichia coli as a cause of NSTI was initially thought to be very rare; however, $E$. coli has recently been recognised as a principle pathogen in monomicrobial NSTIs, and it is common among chronically ill patients with NSTIs $(3,4)$. A versatile pathogen, $E$. coli causes a wide range of extraintestinal diseases, such as urinary tract infection, meningitis and bacteraemia. Until now, however, few reports have analysed the virulence factors of the isolated strains or host susceptibility for E. coli in NSTIs (4). This case report presents a patient with alcoholic liver cirrhosis and NSTI caused by $E$. coli with various virulence genes.

\section{Case Report}

A 75-year-old man was admitted to the emergency department with a 2-day history of constant leg pain and difficulty walking. The patient had a past medical history of acute myocardial infarction and cerebral infarction, and he was receiving treatment for hypertension, diabetes, chronic kidney disease and alcoholic liver cirrhosis (Child-Pugh C).

On emergency admission, the patient appeared unwell and was in pain, with clear consciousness. His temperature was $36.0^{\circ} \mathrm{C}$, blood pressure $82 / 66 \mathrm{mmHg}$, pulse 103 beats $/ \mathrm{min}$ and respiratory rate of 30 breaths $/ \mathrm{min}$. His oxygen saturation was $100 \%$ (on $10 \mathrm{~L} / \mathrm{min}$ oxygen), and he showed signs of acute respiratory distress.

Physical examination revealed purpura, snowball crepitation and cutaneous sensory deficit on the left lower left leg. No injuries were evident on the skin surface (Fig 1 ). The patient was unable to flex or extend his left knee and ankle because of pain; however, the dorsalis pedis pulses were intact. Enhanced computed tomography scans showed inflammation of fat tissue and air inside the extensor digitorum longus muscle, the 
fibularis brevis and the fibularis longus. The Laboratory Risk Indicator for Necrotising Fasciitis (LRINEC) score was 10, based on these values: C-reactive protein $531 \mathrm{mg} / \mathrm{L}$, white blood cells $5.0 \times 10^{3}$ cells $/ \mathrm{mm}^{3}$, haemoglobin $10.5 \mathrm{~g} / \mathrm{dL}$, sodium $126 \mathrm{mmol} / \mathrm{L}$, creatinine $7.09 \mathrm{mg} / \mathrm{dL}$ and glucose $37 \mathrm{mg} / \mathrm{dL}$. These findings suggested that this case had a very high possibility of necrotising fasciitis. Given the clinic, biologic and radiologic factors of this case, a diagnosis of NSTI was made.

After the diagnosis was made $-2 \mathrm{~h}$ after the patient was presented to the emergency department - surgical fasciotomy was performed (Fig. 2 ) to determine the extent of the infection, to assess the need for debridement or amputation and to obtain specimens for Gram staining and culture. First, a 30-cm incision over the most indurated and erythematous area of the left lateral aspect of the calf. On incision, a large amount of fluid and purulence with a foul odour was released. However, macroscopic examination showed no necrosis for the fascia and the muscle tissue of the extensor hallucis longus muscle and extensor digitorum longus muscle. The incision wound was irrigated with large volumes of normal saline but the leg was not amputated.

Tracheal intubation for airway maintenance and mechanical ventilation were performed. The patient was administered meropenem ( 1 g every $12 \mathrm{~h})$, vancomycin hydrochloride ( 1 g every $24 \mathrm{~h})$ and clindamycin (600 mg every $8 \mathrm{~h}$ ). Continuous renal replacement therapy was initiated for metabolic acidosis. His blood pressure was unable to be maintained without administration of a vasopressor agent, so noradrenalin $0.5 \mu \mathrm{g} / \mathrm{kg} / \mathrm{min}$ and vasopressin 2 units/h were initiated.

Subsequently, $8 \mathrm{~h}$ after presentation, blood cultures revealed the presence of a gram-negative bacillus, and polymyxin B immobilised fibre column direct hemoperfusion was performed. In addition, thrombomodulin and antithrombin III were administered for disseminated intravascular coagulation based on these values: platelets $5.7 \times 10^{10} / \mathrm{L}$, d-dimer $4.2 \mu \mathrm{g} / \mathrm{mL}$ and prothrombin ratio $76 \%$.

On evaluation $18 \mathrm{~h}$ after presentation, the purpura of his skin was spreading rapidly. Continuously increasing concentrations were noted for lactic acid (maximum $17 \mathrm{mmol} / \mathrm{L}$ ), blood creatine kinase (maximum $5657 \mathrm{U} / \mathrm{L}$ ) and potassium (maximum $7.7 \mathrm{mEq} / \mathrm{L}$ ). Based on the assumption that his infection was now uncontrollable, the decision was made to amputate the leg. However, $24 \mathrm{~h}$ after presentation, before amputation could begin, the patient died.

The following day, the only pathogen detected in culture samples from the blood, fascia and fluid obtained from the wound was E. coli. Histologic examination showed degenerated fascia and fatty tissue with a bacterial colony present. The susceptibility of the $E$. colistrain to antibiotics was pansensitive. Based on all of these findings, necrotising fasciitis caused by E. coli was diagnosed. Further culture sample analysis revealed that the E. coli strains belonged to sequence type (ST) 127 in multi-locus sequence typing (MLST) and phylogenetic group B2 with various virulence genes, as shown inTable $\mathbf{1}$.

\section{DISCUSSION}

This report presented a case of monomicrobial NSTI caused by E. coli with life-threatening septic shock in a patient with alcoholic liver cirrhosis. In cases of NSTIs typically the chances of survival increase with early surgical intervention, preferably within $6 \mathrm{~h}$ on infection onset $(5,6)$. In this case, however, despite prompt incision and drainage of the infected area within $2 \mathrm{~h}$, it was not possible to save the patient. The reason for this rapid clinical course resulting in mortality was presumed to be the susceptibility between the host and pathogen. Until now not been many reports have investigated the pathogens in NSTIs at the genetic level (3).

\section{Host Factors}

Type 3 NSTIs caused by E. coli are typically seen in immunocompromised patients, such as those with liver cirrhosis, chronic kidney disease and haematologic malignancy (4). For such patients, the potential for a more severe condition to develop is higher and the mortality rate is higher. This degree of increased risk was shown in a report that summarised 18 cases of NSTIs caused by E. coli since 1994, in which $13(73 \%)$ of the patients died (4). 
The patient in this case had alcoholic liver cirrhosis (Child-Pugh C). It is well known that patients with liver cirrhosis are compromised immunologically, a state that often leads to bacteraemia and sepsis by the intestinal-portal route and that results in a high rate of morbidity and mortality $(7,8)$. The main reasons for these outcomes in this patient population are intestinal bacterial overgrowth, bacterial translocation caused by disruption of enteric barrier and dysfunction of hepatic and systemic immune system (8). These factors result in an increase of the risk of bacteraemia and sepsis in patients with cirrhosis.

\section{Pathogen Factors}

Pathogenesis and epidemiology of NSTIs caused by E. coli are not well studied in comparison to uropathogenic E. coli . There are two methods to genetically classify E. coli . The conventional method for classification of $E$. coli is by phylogenetic group. Recently, however, another classification method for $E$. coli has been developed. The MLST method characterises isolates of bacterial species using the sequences of internal fragments of 7 house-keeping genes. In this case, the causative E. coli strain was ST127 in MLST and virulent phylogenetic group B2. Recently, ST127 was recognised as a pathogen in urinary tract infection $(9,10)$. This strain was also reported as a pathogen in necrotising enterocolitis in preterm infants (11) and in bloodstream infections (12). What is notable about this strain is that, despite having high antibiotic sensitivity, it possesses many pathogenic genes. However, the pathogenesis of the ST127 strain in the setting of skin infection has yet to be studied.

In this case, it was also possible to identify the genes of the pathogen. Virulence factors such as adhesion, toxins, iron acquisition and invasion were analysed. The virulence patterns of the strains from the skin and soft tissue infection in this patient were similar on whole-genome analysis to strains from uropathogenic infection and sepsis (13); however, the available epidemiologic data with a large number of $E$. coli strains was insufficient. The virulence factors suspected of having an association with skin and soft tissue infection were cytotoxic necrotising factor 1 and hemolysin $(4,14)$. These toxins were both positive in this patient, and were reported in other cases to trigger inflammation during infection $(15,16)$ as a result of interactions with neutrophils and monocyte-derived dendritic cells. The patient in this case had a weakened immune system caused by liver cirrhosis, which may have altered the appropriate immune response to interaction with these toxins.

\section{CONCLUSION}

In conclusion, NSTI caused by E. coli monomicrobial infection is an extremely severe condition with high mortality. The reason for this degree of severity and mortality may be the susceptibility between host and pathogen. Further study is needed to understand the pathogenesis of $E$. coli during skin and soft tissue infection, especially for cases of necrotising fasciitis in patients with cirrhosis.

\section{ACKNOWLEDGMENT}

We would like to thank enago (www.enago.jp) for English language editing.

\section{CONFLICT OF INTEREST}

None declared.

\section{AUTHOR CONTRIBUTIONS}

MK drafted an initial manuscript. MI and IH reviewed the manuscript at first. KD and KM critically reviewed it. All the authors contributed to management of the patient to writing the manuscript.

\section{ETHICAL APPROVAL}

Published with the written consent of the patient.

\section{CONSENT FOR PUBLICATION}

Written informed consent was obtained from the patient for publication of this case report and its accompanying image. 


\section{FUNDING}

This report did not receive any specific grant from funding agencies in the public, commercial, or not-forprofit sectors.

\section{DATA AVAILABILITY STATEMENT}

Data sharing was not applicable to this article as no datasets were generated or analyzed during the current study.

\section{KEY WORDS}

necrotising soft tissue infections, Escherichia coli, virulence factor, immunocompromised patients, multilocus sequence typing

\section{KEY CLINICAL MESSAGE}

NSTI caused by E. coli monomicrobial infection is an extremely severe condition with high mortality. It is important to know both pathogen virulence factors and host susceptibility factors to recognize the severity of the disease.

\section{REFERENCE}

1. Anaya DA, Dellinger EP. Necrotizing soft-tissue infection: diagnosis and management. Clin Infect Dis 44(5):705-710, 2007.

2. Sarani B, Strong M, Pascual J, Schwab CW. Necrotizing fasciitis: current concepts and review of the literature. J Am Coll Surg 208(2):279-288, 2009.

3. Grimaldi D, Bonacorsi S, Roussel H, et al. Unusual "flesh-eating" strain of Escherichia coli. J Clin Microbiol 48(10):3794-3796, 2010.

4. Gallois C, Hauw-Berlemont C, Richaud C, Bonacorsi S, Diehl JL, Mainardi JL. Fatal necrotizing fasciitis due to necrotic toxin-producing Escherichia coli strain. In: New Microbes New Infect 8:109-112, 2015.

5. Bucca K, Spencer R, Orford N, Cattigan C, Athan E, McDonald A. Early diagnosis and treatment of necrotizing fasciitis can improve survival: an observational intensive care unit cohort study. ANZ J Surg 83(5):365-370, 2013.

6. Hadeed GJ, Smith J, O'Keeffe T, et al. Early surgical intervention and its impact on patients presenting with necrotizing soft tissue infections: A single academic center experience. J Emerg Trauma Shock 9(1):2227, 2016.

7. Foreman MG, Mannino DM, Moss M. Cirrhosis as a risk factor for sepsis and death: analysis of the National Hospital Discharge Survey. Chest 124(3):1016-1020, 2003.

8. Bonnel AR, Bunchorntavakul C, Reddy KR. Immune dysfunction and infections in patients with cirrhosis. Clin Gastroenterol Hepatol 9(9):727-738, 2011.

9. Gibreel TM, Dodgson AR, Cheesbrough J, Fox AJ, Bolton FJ, Upton M. Population structure, virulence potential and antibiotic susceptibility of uropathogenic Escherichia coli from Northwest England. J Antimicrob Chemother 67(2):346-356, 2012.

10. Banerjee R, Johnston B, Lohse C, et al. The clonal distribution and diversity of extraintestinal Escherichia coli isolates vary according to patient characteristics. Antimicrob Agents Chemother 57(12):59125917, 2013.

11. Ward DV, Scholz M, Zolfo M, et al. Metagenomic sequencing with strain-level resolution implicates uropathogenic E. coli in necrotizing enterocolitis and mortality in preterm infants. Cell Rep 14(12):2912-2924, 2016. 
12. Ciesielczuk H, Jenkins C, Chattaway M, et al. Trends in ExPEC serogroups in the UK and their significance. Eur J Clin Microbiol Infect Dis 35(10):1661-1666, 2016.

13. Ranjan A, Shaik S, Nandanwar N, et al. Comparative genomics of Escherichia coli isolated from skin and soft tissue and other extraintestinal infections. mBio 8(4), 2017.

14. Rippere-Lampe KE, Lang M, Ceri H, Olson M, Lockman HA, O'Brien AD. Cytotoxic necrotizing factor type 1-positive Escherichia coli causes increased inflammation and tissue damage to the prostate in a rat prostatitis model. Infect Immun 69(10):6515-6519, 2001.

15. Rippere-Lampe KE, O'Brien AD, Conran R, Lockman HA. Mutation of the gene encoding cytotoxic necrotizing factor type $1(\operatorname{cnf}(1))$ attenuates the virulence of uropathogenic Escherichia coli. Infect Immun 69(6):3954-3964, 2001.

16. Gall-Mas L, Fabbri A, Namini MRJ, Givskov M, Fiorentini C, Krejsgaard T. The bacterial toxin CNF1 induces activation and maturation of human monocyte-derived dendritic cells. Int J Mol Sci 19(5), 2018.

\section{FIGURE LEGENDS}

Fig. 1. Physical examination revealed purpura, snowball crepitation and cutaneous sensory deficit on the left lower left leg, but no injuries were evident on the skin surface.

Fig. 2. Surgical fasciotomy was performed with a $30-\mathrm{cm}$ incision over the most indurated and erythematous area of the left lateral aspect of the calf. However, the fascia and the muscle tissue beneath the fascia of the extensor hallucis longus muscle and extensor digitorum longus muscle were pink, and not necrotic.

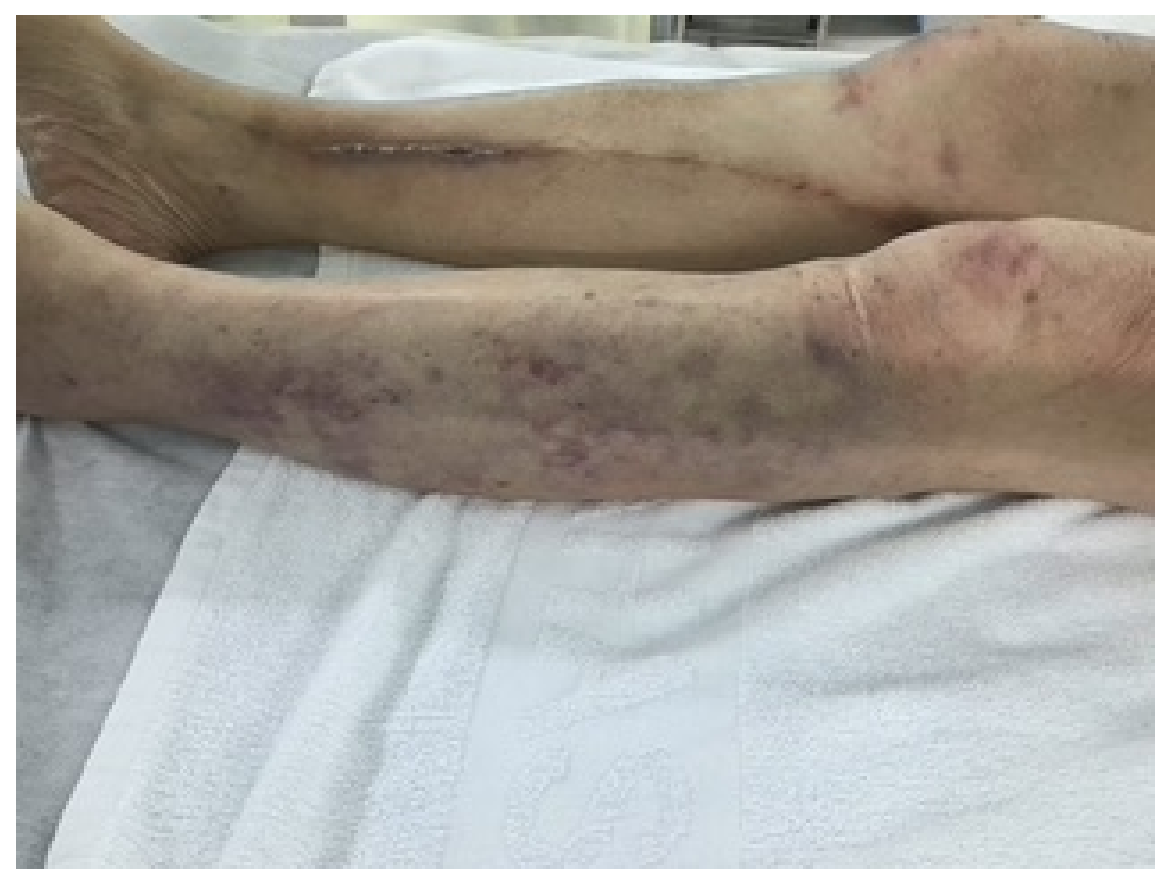




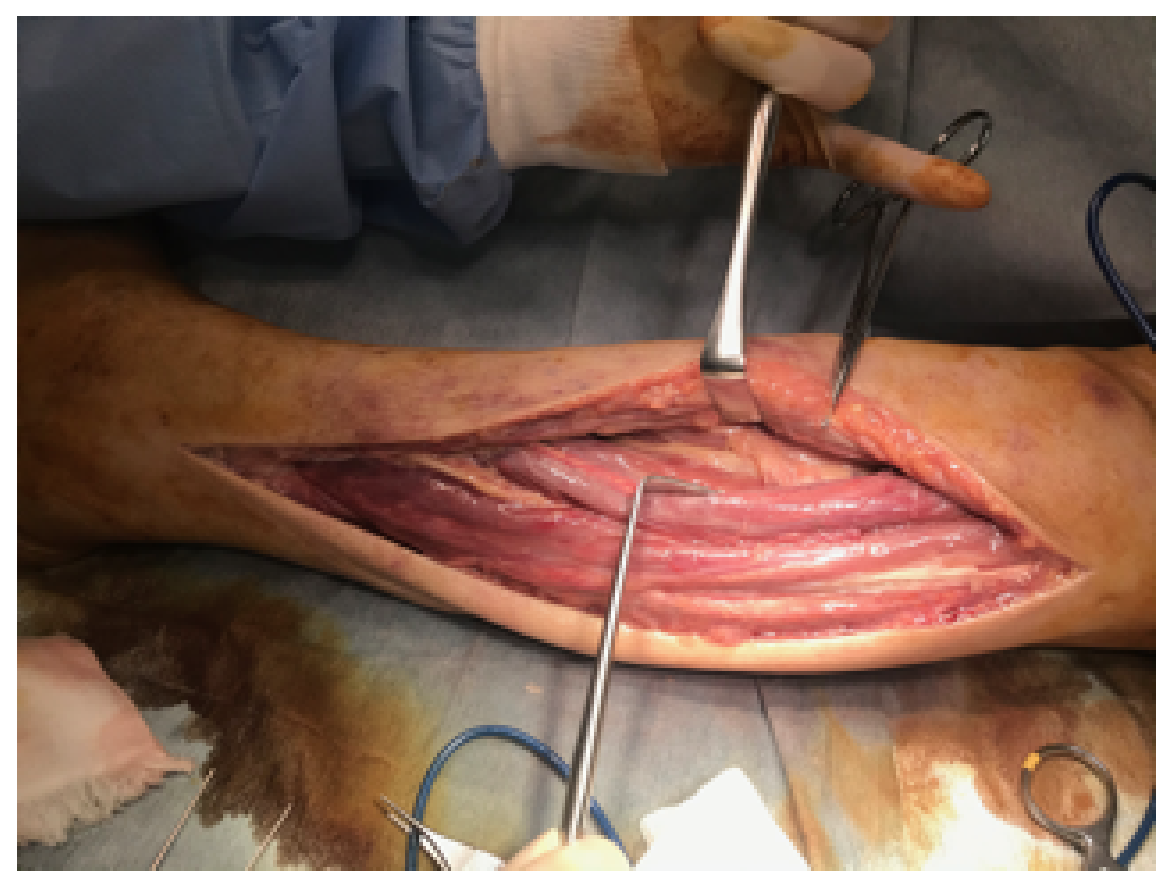

\section{Hosted file}

Table1_Ec_virulence gene_.docx available at https://authorea.com/users/416823/articles/ 524209-fatal-necrotising-soft-tissue-infection-caused-by-escherichia-coli-in-alcoholicliver-cirrhosis 